\title{
Le Divorce Des Pasteurs En Milieu Evangelique Ivoirien : Approche Socio-Anthropologique D’un Phenomene Desintegrateur A Travers Le Cas Des Communautes « Assemblees De Dieu » Et « Ministere International De La Revelation » De Yopougon, Abidjan, Côte-d'Ivoire
}

\author{
N'dri Kouadio Patrice \\ Université Alassane Ouattara de Bouaké, Côte d’Ivoire
}

doi: 10.19044/esj.2017.v13n8p302 URL:http://dx.doi.org/10.19044/esj.2017.v13n8p302

\begin{abstract}
In evangelical communities, divorce is prohibited and a pastor is seen as a model, a spiritual guide and, above all, a guarantor of the Christian faith and morality. From this point of view, the breakdown of the marriage bond of a pastor with his wife undermines the cohesion and stability of the communities under his tutelage. This is because divorce is considered in Christian circles as a transgressive and anomic act. This article is a comprehensive approach of the divorce of pastors. It also shows its explanatory factors using a case study of two localities, the Assemblies of God Church and the International Ministry of the Revelation of Yopougon. The study was carried out using a socio-anthropological approach combined with both qualitative and quantitative methods.

Based on the result of this study, the divorce of pastors is explained by several factors. The most essential include: the tensions and the recurring disputes in the couple; the continued infidelity of the spouses; the lack of forgiveness and reciprocal acceptance of spouses in case of error; the subtle flight of one of the spouses of the household, and so on. Although they are human guides and shepherds, pastors who have succumbed to these situations have destroyed the sacred bond of their marriage. These kinds of behavior of the evangelical guides have led to the regression of the social and political functioning of the Christian communities. Divorce, however, has become a symbolic act of destruction of socio-religious bonds while tarnishing the image of the evangelical world.
\end{abstract}

Keywords: Divorce, pastors, evangelical churches, Ivory Coast. 


\section{Résumé}

Dans les communautés évangéliques, le divorce est un interdit et le pasteur représente un modèle, un guide spirituel et surtout le garant de la foi et de la morale chrétienne. De ce point de vue, la rupture du lien de mariage avec son épouse met en mal la cohésion et la stabilité des communautés sous sa tutelle ; car le divorce est considéré en milieu chrétien comme un acte transgressif et anomique. Ce présent article se veut donc une approche compréhensive du divorce des pasteurs et ses facteurs explicatifs à travers le cas de deux localités-témoins à savoir : l'Eglise des Assemblées de Dieu et le Ministère International de la révélation de Yopougon. La réalisation de cette étude a mobilisé comme méthode l'approche socio anthropologique alliée aux méthodes qualitatives et quantitatives. Comme résultat, le divorce des pasteurs s'explique par plusieurs facteurs dont les plus essentiels sont: les tensions et les disputes récurrentes dans le couple ; l'infidélité continue des époux ; le manque de pardon et d'acceptation réciproque des conjoints en cas d'erreur; la fuite subtile de l'un des conjoints du foyer, etc. Bien qu'ils soient des guides et des bergers humains, les pasteurs succombent à ces situations et détruisent le lien sacré de leur mariage. Ces genres de comportements des guides évangéliques conduisent à la régression du fonctionnement social et politique des communautés chrétiennes. Ainsi, le divorce devient un acte symbolique de destruction des liens socio religieux tout en ternissant l'image du monde évangélique.

Mots clés: Divorce, pasteurs, Eglises évangéliques, Côte d’Ivoire.

\section{Introduction}

Depuis 1990, année où le vent démocratique a soufflé sur la Côte d'Ivoire, on assiste à la liberté d'expression ${ }^{7}$ religieuse sous forme d'effervescence et d'expansion de nouveaux mouvements religieux pentecôtistes (Guiblehon, 2012 : 116). Cette liberté d'expression religieuse a favorisé la naissance de plusieurs ministères et missions internationales. Au niveau des couples chrétiens, les conjoints se soumettent de moins en moins aux instructions des guides religieux et acceptent difficilement leurs conseils en cas de situation problématique. Du coup, on assiste à des tensions dans ces couples chrétiens, qui continuent de provoquer des effets dans le fonctionnement des communautés. Car ces inconforts conduisent parfois « à la rupture de lien de mariage au nombre desquels on retrouve des pasteurs,

\footnotetext{
${ }^{7}$ Entendons par liberté d'action le fait de poser un acte sans contrainte et surtout sans rencontrer d’opposition. En milieu évangélique les pasteurs ont la liberté de créer des Eglises sans être contraint par qui ou par quoi que ce soit. Cette liberté d'action qui se vit comme un libertinage pousse certains pasteurs à agir selon leur gré pourvu que leurs objectifs soient atteints.
} 
des prophètes, des évangélistes... bref, des guides spirituels » ${ }^{8}$. Dans cet article le cas des pasteurs a retenu notre attention.

En effet, considérés sous l'étiquette de bergers ou de guides spirituels ou religieux, les pasteurs sont "les portes paroles de Dieu" dans les communautés chrétiennes. Ils sont chargés d'annoncer, d'enseigner, de guider, d'orienter "le troupeau" pour que le projet de Dieu pour cette entité aboutisse $^{9}$ (N'Dri, 2015). Pour mener à bien cette mission religieuse, certains pasteurs passent par une formation théologique et sacerdotale qui leur donne accès à des connaissances profondes ${ }^{10}$ (Balandier Georges, 1996) et à des capacités, tandis que d'autres se contentent de leur pouvoir ou capacité dite surnaturelle qu'ils utilisent soit pour agiter, soit pour faire tomber des personnes qu'ils dénomment "onction" "11". «La formation théologique, pour ceux qui en font, constitue une période pendant laquelle ces pasteurs apprennent les fondements doctrinaux dans des écoles religieuses en vue d'avoir les qualifications socio religieuses propices à l'exercice de leur fonction de pasteur ${ }^{12}$. De ces positions ci-dessus l'acquisition cognitive sinon la formation en théologie représente un atout indispensable qui renforce le caractère sacré de leur fonction auprès des fidèles dont ils ont la charge. A cet effet, les pasteurs sachant comprennent la théologie pastorale relative au divorce comme interdit religieux et prennent des dispositions pour son application dans les communautés chrétiennes.

Cependant, aujourd'hui nous constatons en Côte d'Ivoire que des pasteurs d'Eglises évangéliques divorcent et se remarient avec d'autres partenaires, bien que le divorce soit un acte proscrit par la doctrine

${ }^{8}$ Propos recueilli auprès du pasteur Zouzoua Pierre de la Mission Evangélique des Rachetés de Dieu.

${ }^{9}$ N'Dri K. P. (2015). Le divorce en milieu évangélique et ses conséquences sociales : cas de cinq communautés évangéliques de Yopougon-Côte d'Ivoire, thèse de doctorat, Université Alassane Ouattara de Bouaké, p 320.

${ }^{10}$ Balandier Georges (1996). "le sacré par le détour des sociétés de la tradition” in Cahiers internationaux de sociologie, vol 100, version électronique consulté le 29/04/2015, PUF, Paris, p 10

11 "Onction" est un terme qui provient du mot Latin unctio qui signifie « action d'oindre ». Contrairement au sens théologique qui stipule que l'onction est un acte liturgique qui fait partie de certains sacrements chrétiens (confirmation, extrême-onction, etc.), ce groupe de pasteurs appellent onction la capacité qu'ils détiennent et qui leur permettent d'agiter ou de faire tomber une tierce personne au cours des séances de prières. Ainsi un pasteur qui par sa prière provoque ces manifestations mentionnées ci-dessus est dit avoir l'onction en sa possession. Pour eux, ces indicateurs constituent la confirmation de ce pouvoir qu'ils disent avoir reçu de Dieu. Autrement dit, ces manifestations de capacité par des vives agitations provoquées lors des prières exorcistes sont les signes que Dieu leur a donné l'onction et qu'ils ont le droit de l'exercer comme bon leur semble.

${ }^{12}$ Propos recueilli auprès du pasteur Yao N'guettia, conseillé conjugale et président de l’ONG famille. 
évangélique. Dans le milieu évangélique ivoirien la rupture ${ }^{13}$ du lien matrimonial peut occasionner des sanctions disciplinaires allant jusqu'à l'excommunication. L'application de cette disposition disciplinaire se justifie par le fait que la désobéissance de la norme sociale en tant que règle de conduite occasionne des sanctions appliquées par la communauté contre la victime $^{14}$ (Piras Mauro, 2004). Pour le cas spécifique des pasteurs évangéliques les sanctions varient d'une dénomination à une autre et dépendent des facteurs justificatifs du divorce, du contexte social évoqué, de la représentation que les pasteurs ont d'eux-mêmes et de la perception dont ils sont objets dans la conscience des collectivités religieuses. C'est pourquoi, certaines communautés acceptent les pasteurs divorcés, d'autres les chassent et d'autres encore les suspendent de leur charge pastorale en leur donnant le statut de simple fidèle. Dans ce contexte socio religieux confuse, le mariage qui est aussi bien dans la tradition africaine que dans celle judéochrétienne, un instrument de pérennisation de l'espèce humaine ${ }^{15}$ (Chimoun Mosé, 2004) et un acte conduisant à un lien sacré perd progressivement ses valeurs sociales et religieuses.

Le comportement de divorce des pasteurs, puisque garant de la foi et de la morale chrétienne, a des implications intra et extra communautaires. Au-delà de ce qui précède, le sexe en tant que sujet tabou et organe sacré dans la conception africaine ${ }^{16}$ est désacralisé.

Cet article qui s’inscrit dans une perspective socio-anthropologique et religieuse, se donne comme objectif principal d'analyser les registres mobilisés pour justifier le divorce des pasteurs évangéliques d'une part ; et d'autre part d'appréhender les implications à la fois communautaires et sociales du phénomène du divorce des pasteurs à travers le cas des Eglises des Assemblés de Dieu (AD) et du Ministère International de la Révélation (MIR) de la Commune de Yopougon.

La thèse soutenue est la suivante: le divorce des pasteurs qui se justifie par les situations d'inconfort dans le couple est un phénomène qui désintègre l’ordre communautaire et son environnement social.

\footnotetext{
${ }^{13}$ Jésus Christ au sujet du divorce a dit « au commencement il n'en était pas ainsi. Mais je vous dis que celui qui répudie sa femme,... et qui en épouse une autre, commet un adultère » Matthieu 19v8-9.

${ }^{14}$ Piras Mauro T. (2004). «Les fondements sociaux de l'agir normatif chez Durkheim et Weber : le rôle du sacré » in Archives des Sciences Sociales des Religions, 127, p 154.

${ }^{15}$ Chimoun Mosé (2004). «transgressions des tabous sexuels dans les romans féministes de l'Afrique de l'Ouest, du centre et du monde germanophone » in Langues et Littératures, Université Gaston berger de Saint-Louis, Nº8, Sénégal, p 65.

${ }^{16}$ Idem p 70
} 
Au plan méthodologique, nous avons eu recours aux entretiens avec des pasteurs divorcés issus de ces deux (02) dénominations ${ }^{17}$ évangéliques de Yopougon; d'anciens d'église ; de responsables de jeunes et de femmes ainsi que des fidèles. Les investigations ont été réalisées à partir de guides d'entretiens semi-directifs et des récits de vie. L'analyse des données recueillies a permis de mettre en lumière les registres justificatifs du divorce et ses implications sociales. Car les représentations des acteurs locaux sont des éléments indispensables à toute compréhension du fait social ${ }^{18}$ (Oliver de Sardan, 2003). L’éclairage de ces données s’est effectué à l'aide de la méthode compréhensive de Max Weber et de l'analyse de contenu. Les théories de la résilience et de la justification de Boltanski Luc (2001) ont renforcé les analyses à cause des multiples facettes que comporte cette investigation.

Soulignons par ailleurs que la question du sexe chez l'africain est rangé dans le registre du tabou. De ce fait, il s'est posé un problème épistémologique lors de nos interviews. Nous l'avons perçu par la réticence de certains enquêtés qui lors des échanges ont éprouvé des gènes à s'exprimer sur les questions liées aux comportements intimes. D’autres refusent catégoriquement les questions surtout liées à la sexualité. Cependant, ces difficultés ont été surmontées à partir de notre expérience de terrain qui a contribué à faciliter la dynamique du rapport enquêteur-enquêté.

Ce comportement lié au divorce dans les couples n’a pas laissé indifférent la communauté du monde des écrivains et chercheurs qui en ont fait déjà l'objet de leur multiple réflexion: Gascon E., 2001; Cardier-Rey, 2004; Ako G., 2013; Murray J., 1992; Kosongo M. P. \& Kinable J., 2010; Aman K. L, 2006; Atsain A. J. P. et al., 1997; Bale M., 1988, etc. De ces différents ouvrages il nous revient que le divorce est un mal social dans l'ensemble de la société et un interdit doctrinal dans les communautés religieuses. Ce comportement aux origines diverses est également un facteur de mutation aussi bien dans les familles, les Eglises et leur environnement sociétal que dans la société en général.

Notre préoccupation de recherche est la suivante: comment expliquer les justifications liées au divorce de pasteurs et leurs implications communautaires et sociales dans un milieu où cet acte est proscrit ? De cette question principale découle trois préoccupations subsidiaires: quels sont les fondements doctrinaux du divorce chez les évangéliques de Côte d’Ivoire? Quels sont les facteurs justificatifs du divorce des pasteurs chez les

\footnotetext{
${ }^{17}$ Nous avons retenus une communauté par dénomination évangélique concernée ; ce sont : les AD de Yopougon Zones industrielles et la communauté MIR de Yopougon Toit rouge. ${ }^{18}$ Oliver de Sardan J.P. (2003). L'enquête socio-anthropologique de terrain: synthèse méthodologique et recommandations à usage des étudiants, études et travaux $\mathrm{N}^{\circ} 13$, LASDEL, Niamey, p 37.
} 
évangéliques? Quelles sont les implications communautaires et sociales de ce comportement de ces pasteurs?

Pour mener à bien notre analyse en vue de répondre objectivement à ces préoccupations ci-dessus, nous allons considérer deux cas de divorce de pasteurs dont un dans chaque communauté choisie. Cette étude s’articule autour de trois mouvements. Il s’agit d'abord de faire un aperçu sur les fondements doctrinaux du divorce chez les évangéliques de Côte d’ivoire, ensuite de relever les facteurs justificatifs du divorce des pasteurs, et enfin d'analyser les implications communautaires et sociales de ce comportement.

\section{Les fondements doctrinaux du divorce chez les évangéliques de Côte d'Ivoire}

La doctrine du divorce étant dépendante, à l'évidence, de celle du mariage $^{19}$ (Murray John, 1992) nous ne pouvons analyser les fondements doctrinaux du divorce sans ceux du mariage. Quelques données bibliques seront évoquées pour l'éclairage de nos argumentations dans cette section. Ce faisant, nous allons aborder brièvement les fondements doctrinaux du mariage chez les évangéliques.

\section{I-1 Les fondements doctrinaux du mariage}

Généralement perçu comme l'union de deux personnes, le mariage chez les évangéliques concerne deux personnes de sexe opposé, c'est-à-dire l'homme et la femme. Pour eux ce modèle matrimonial est une prescription biblique dont le récit se trouve dans le livre de la Genèse ${ }^{20}$. Nous comprenons par ce récit que le mariage dans ses fondements bibliques et doctrinaux est une union monogame et hétérosexuelle. Pour les évangéliques les unions visent plusieurs buts dont la procréation humaine, le peuplement et la gouvernance de la terre par l'Homme ${ }^{21}$. Il importe de préciser que chez les protestants évangéliques le mariage n'est pas un sacrement comme c'est le cas chez les catholiques. Toutefois, cette institution est régit par des rites sacrés et le lien qui en découle est indestructible ${ }^{22}$, même si des prescriptions

\footnotetext{
${ }^{19}$ Murray John (1992). Le divorce : les données bibliques, Collection Alliance, les Editions Sator, Grande Bretagne, p 14.

${ }^{20}$ Dieu créa l’homme et la femme. Dieu les bénis, et Dieu leur dit: soyez féconds, multipliez, remplissez la terre, et l'assujettissez... c'est pourquoi l'homme quittera son père et sa mère, et s'attachera à sa femme, et ils deviendront une seule chair, Genèse 1v27-28/ 2v24 version Louis Ségond.

${ }^{21}$ L’Homme ici désigne l'ensemble de la créature humaine à qui l'ordre est donné de se multiplier en vue du peuplement et de la gouvernance de la terre.

${ }^{22}$ Cette indestructibilité du lien de mariage est attestée par la prescription biblique qui affirme ceci « que l’homme donc ne sépare pas ce que Dieu a joint » Marc 10v9.
} 
bibliques donnent l'occasion aux conjoints d'envisager la rupture dans certains cas tel que l'infidélitée ${ }^{23}$ et les actes sexuels en dehors du conjoint.

Dans la perspective de la théologie protestante, les unions requièrent des conjoints engagement libéral susceptible de les mener à une vie communautaire et à l'union sexuelle. De par cet engagement, les conjoints unis, à la lumière des prescriptions bibliques doctrinales, sont désormais liés par un lien indestructible que seule la mort rompre.

Au niveau de la Commune de Yopougon, les chrétiens évangéliques se marient sur la base des prescriptions bibliques et dans le respect du modèle hétérosexuel. Cette hétérosexualité observée dans les contractions des unions représente le prototype de mariage que l'Eglise a défendu jusqu'aujourd'hui. Généralement la manifestation des mariages dans les communautés chrétiennes obéissent, selon le pasteur Y. S. ${ }^{24}$, à un processus comportant trois étapes: culturelle (ou anthropologique) ; civil et religieux. Pour lui ces deux premières étapes, chez les évangéliques, sont obligatoires pour la suite du processus du mariage. C'est pourquoi, ajoutera-t-il, les conjoints pour se conformer à la doctrine matrimoniale évangélique se soumettent aux différentes règles et principes. Ces principes, explique-t-il, exigent du jeune prétendant une dot à payer et d'autres rites complémentaires tels que des libations à exécuter. Cependant, les parents de ce dernier étant chrétiens procèdent par des négociations pour obtenir de la future belle famille l'annulation des rites qui sont à leurs yeux en contradiction avec les prescriptions bibliques. Dans ce contexte, le pasteur Yao mentionne par exemple les sacrifices d'animaux, les libations,... comme éléments culturels qui ne concordent pas avec les habitudes matrimoniales chez les évangéliques.

L'Eglise dans son ensemble et particulièrement chez les évangéliques, les leaders religieux considèrent la satisfaction des exigences coutumières et civiles comme conditions sine quoi non pour le mariage religieux. Néanmoins pour le droit canonique catholique, le mariage est l'union d'un homme et d'une femme généralement baptisés (SEBO Kouami F., 2014: 47). Ce critère, bien qu'il ne soit pas universel à toutes les dénominations chrétiennes est facultatif chez les évangéliques. Ces unions qui se contractent publiquement sont scellées par l'échange des consentements verbaux et sous forme matériel comme c'est le cas pour le port des anneaux par les conjoints. Ces rites qui se réalisent après un sermon conduit inéluctablement à l'établissement d'un lien sacré. Saint Paul abordant dans cette perspective estime que ce lien sacré qui représente une

\footnotetext{
${ }^{23}$ «Celui qui répudie sa femme, sauf pour infidélité et qui en épouse une autre commet un adultère » Matthieu 19v9.

${ }^{24}$ Y. S., ex-pasteur des AD de la Yopougon zone industrielle.
} 
alliance est indestructible, tant que les conjoints sont en vie ${ }^{25}$. Cette alliance est similaire à celle symbolique unissant Dieu et le peuple de la Bible (Israël), et celle existant entre l'Eglise et le Christ, d'autre part. De ce fait, le divorce devient un interdit puisqu'il représente le brisement de ce lien, selon les prescriptions bibliques des communautés évangéliques.

Voilà brièvement présenté les fondements doctrinaux du mariage chez les évangéliques en Côte d'Ivoire.

Par ailleurs, ajoutons que dans les communautés chrétiennes le mariage peut créer des réseaux sociaux dont les limites s’étendent au-delà des Eglises. Ainsi, il s'avère un moyen de conciliation et surtout un facteur de cohésion sociale, de brassages culturels, ethniques et de rapprochement aussi bien des familles, des peuples, des communautés religieuses, etc.

Mais qu'en est-il des fondements doctrinaux du divorce et surtout que disent les prescriptions bibliques?

\section{I-2 Les fondements doctrinaux du divorce}

Réalité sociale, le divorce est généralement perçu comme la rupture du lien entre deux personnes précédemment unies par le mariage. Chez les chrétiens, rappelons le, cette rupture concerne uniquement le lien existant entre un homme et une femme que Dieu a joint. Au nombre des textes bibliques qui le justifient deux ont retenu notre attention. Il s'agit de Deutéronome 24v1-4 de l'ancien testament et celui de Matthieu 19v6-9 du nouveau testament. Le texte de Deutéronome 24v1-4 dit ceci:

«(v1) Lorsqu'un homme aura pris et épousé une femme qui viendrait à ne plus obtenir sa faveur, parce qu'il aura trouvé en elle quelque chose d'inconvenant, il écrira pour elle une lettre de divorce et, après la lui avoir remise en main, il la renverra de sa maison (v2.). Elle sortira de chez lui, s'en ira et pourra devenir la femme d'un autre homme (v3). Si ce dernier cesse de l'aimer, écrit pour elle une lettre de divorce et, après la lui avoir remise en main, la renvoie de sa maison; ou bien, si ce dernier homme qui l'a prise pour femme vient à mourir, (v4) alors, le premier mari qui l'avait renvoyée ne pourra pas la reprendre pour femme après qu'elle a été souillée, car c'est une horreur devant l'Eternel, et tu ne chargeras pas d'un péché le pays que l'Eternel ton Dieu, te donne pour héritage $»{ }^{26}$.

Ce texte décrit la procédure à suivre pour rompre d'avec son conjoint selon la torah. Ici la raison qui peut occasionner la rupture du lien de mariage

\footnotetext{
${ }^{25}$ Romain 7v2 version Louis Ségond

${ }^{26}$ Ce texte de Deutéronome 24 v 1-4 est issu de la version de la Bible dite à la Colombe pour les traductions protestantes.
} 
est désignée par l’expression "quelque chose d'inconvenant". Cette raison dont le contenu n'est pas explicite donne l’opportunité à l'époux de renvoyer son épouse en lui remettant une lettre de divorce. Pour John Murray (1992) cette manière d’agir faisait office de norme législative en ce qui concerne la question du divorce et de la répudiation chez les Juifs de cette époque. Comme conséquence l'épouse divorcée a la possibilité de se remarier mais ne peut plus reprendre la vie communautaire avec le premier homme qui l'avait prise en aversion, même si ce dernier le souhaiterait.

La question de divorce ressurgît à l'époque de Jésus-Christ lorsque les pharisiens l'interrogèrent à propos. Il répondit ceci lors d’une séance d'enseignement public:

«Que l'homme donc ne sépare pas ce que Dieu a joint. Pourquoi donc, lui dirent-ils, Moïse a-t-il prescrit de donner à la femme une lettre de divorce et de la répudier? Il leur répondit: c'est à cause de la dureté de votre cœur que Moïse vous a permis de répudier vos femmes; au commencement, il n'en était pas ainsi. Mais je vous dis : quiconque répudie sa femme, sauf pour infidélité, et en épouse une autre, commet un adultère ${ }^{27}$.

L’auteur de ces propos donne ici la position de Jésus concernant le mariage et le divorce. Ainsi pour Jésus, le lien matrimonial établi par Dieu est indestructible. Par conséquent l'homme ne doit pas le rompre. L'autorisation de divorce ordonnée par Moïse se justifie par la dureté du cœur de l'homme, c'est-à-dire son refus de pardonner et d'accepter son exépouse comme telle en vue d'une vie sociale harmonieuse. Toutefois, selon saint Matthieu les implications du divorce en dehors des cas d'infidélité se résument au péché d’adultère.

Dans les communautés AD et MIR, les autorités religieuses recourent à ces prescriptions pour traiter les cas de divorce en leur sein. Mais des justifications évoquées dans ces communautés relèvent de l'ordre social et coutumier des peuples surtout des habitudes communautaires en conflit avec les habitudes modernes. De ce fait, on se demande pourquoi, malgré ces connaissances doctrinales dispensées dans les communautés par des leaders proscrivant le divorce, les leaders eux-mêmes, en tant que garants spirituels et moraux de ces enseignements arrivent à la rupture ? Répondre à cette interrogation nous amène à aborder les raisons liées au divorce des pasteurs en milieu évangélique.

${ }^{27}$ Matthieu 19 v 6-9, version Louis ségond. 


\section{Les facteurs justificatifs de divorce des pasteurs des Eglises évangéliques}

Bien que le divorce soit un concept commun en milieu évangélique, les justifications et les représentations varient d'une dénomination à une autre. De nos interviews dans les Eglises il nous revient que, celles qui obéissent à une organisation nationale dont les $\mathrm{AD}$, les pasteurs sont soumis à des principes bien élaborés qui régularisent la conduite des pasteurs et responsables des communautés. Chez les AD par exemple, l'un des principes internes stipule que, tout pasteur qui divorce d'avec son épouse est automatiquement suspendu de sa fonction de pasteurs et radier. Ainsi cette mesure rentre en vigueur juste après le divorce. Si les protagonistes se réconcilient et annulent le divorce, la communauté les réintègre et les rétablit dans leur fonction pastorale. A contrario, les divorcés suspendus sont radiés des effectifs de cette église. Malgré cela, comment les pasteurs arrivent-ils au divorce?

Les investigations menées dans les communautés ciblées et surtout les entretiens avec les pasteurs, les responsables de départements et des fidèles évangéliques mettent en relief les cas de divorce des pasteurs. En réalité le divorce est un phénomène qui désintègre les couples pasteurs et dont les implications rejaillissent sur la communauté et son environnement. A cet effet, nous avons observé deux cas de figure. Dans le premier cas décrit ci-dessous qui est moins récurrent, c'est le départ de l'épouse organisé par ses propres parents en vue de lui trouver un autre point de chute. Pour mieux comprendre ce cas de figure appuyons nous sur le cas du premier guide religieux appartenant à la première dénomination (AD) choisie pour cette étude.

- Cas de Y. S., ex-pasteur des AD radié des AD de Côte d'Ivoire après son divorce d'avec son épouse $K$. $J$.

L'Eglise des AD regroupe plusieurs communautés dont celles de Yopougon zone industrielle où Y. S était le pasteur principal. Il est l'un des pasteurs qui a marqué cette église, puisqu'il a contribué à son extension et à l'ouverture de plusieurs annexes. Cependant, cette belle aventure va connaître une mauvaise tournure avec le divorce de ce pasteur d'avec son épouse $\mathrm{K} \mathrm{J}$.

En effet, Y. S., pasteur aux AD depuis plus d'une décennie avait pour épouse $\mathrm{K}$ J. Quelques mois après leur union en 2002 il eut des tensions dans leur foyer. La raison évoquée est la suivante : K J., diplômée et ayant des parents aisés désire exercer une profession en dehors du cadre ecclésiastique. Ce désir qui n'a pas l'assentiment de son époux pasteur est l'objet principal de leurs disputes quotidiennes. Devant l'intransigeance de son époux, K. J. décida de déserter le foyer de manière subtile en 2003. Pour ce faire, elle réussit à obtenir une permission de la part de son époux pour aller rendre 
visite à ses parents. Une fois au domicile parental elle y restera définitivement. La belle famille ayant constaté que le pasteur Y.S. n’a ni réagit ni rendu visite et même interpelé la belle famille, pris ses responsabilités après l'échec des tentatives de réconciliation organisées par les membres de la dite communauté. Après une période de deux ans, la belle famille autorisa $\mathrm{K} \mathrm{J}$, à l'insu des responsables des $\mathrm{AD}$, à rejoindre les proches parents à Paris en France et par la suite elle se retrouva en Allemagne où elle obtint une autorisation de dissolution de son mariage avec Y. S. et se remaria.

Le clergé des $\mathrm{AD}$ informé du départ de $\mathrm{K}$. J. donna les moyens suffisants à Y.S., avec l'accord des membres de la belle famille, à rejoindre son épouse en Allemagne pour règlement des différents qui les opposaient. Le nécessaire pour le voyage avaient été pourvu. Certes, Y. S. munit cette somme importante d'argent effectua un long voyage en 2012 faisant croire à ses responsables hiérarchiques qu'il avait rencontré son épouse. Or c'était des faussetés car il préparait son point de chute puisqu'il était conscient de sa radiation certaine.

K. J. de retour d'Allemagne en Côte d'Ivoire en août 2014, déposa sa bague de mariage avec le pasteur Y. S. au siège des $\mathrm{AD}$ comme preuve de sa rupture d'avec ce dernier. Le clergé qui se rendit compte que Y. S. leur a conté des faussetés ne tarda pas à prononcer sa radia le 14 septembre 2014, après une rencontre pastorale. Cette radiation selon eux est justifiée par le fait que, Y.S. manque de crédibilité et a fait usage de faut en leur donnant des informations non fondées sur son épouse en voyage. Pire encore, son épouse ayant désertée le foyer et obtenu le divorce, Y. S. n’est plus considéré comme un modèle et garant de la morale religieuse. Dès lors, il fut excommunié et radié définitivement de l'Eglise des $\mathrm{AD}$. Comme le souligne bien Mlle Solange, l'une de nos enquêtées "l'une des raisons du divorce de $Y$. S. et de K. J. est que, K. J. ayant fait de grandes études ne voulait pas abandonner ses diplômes pour venir servir auprès de son mari comme responsable d'Eglise ${ }^{28}$.

Une fois expulsé, le pasteur Y S. devint objet de division au sein de sa communauté d’origine. Un groupe le suivi dans sa déchéance tandis que l'autre groupe resté dans ladite communauté refusa d'appuyer financièrement cette église. Le pasteur aidé par ses partisans fonda son église dont le nom est "église évangélique la maison de la gloire », lieu où il est considéré comme le président fondateur et pasteur principal.

Du coup, cette communauté AD vît son effectif chuté brutalement avec le départ progressif de certains de ses membres vers d'autres horizons.

${ }^{28}$ Mlle Solange, chantre de la communauté AD de Yopougon Zone industrielle, Côte d'Ivoire. 
Le pasteur divorcé qui avait senti sa radiation certaine a eu le temps nécessaire pour vider la caisse de la communauté avec l'implication de ses proches avant sont départ. Cet acte qui s’avère "une infraction financière" a affaibli les capacités financières de cette communauté.

Yopougon le 18 mars 2016

En définitive, nous retenons que chez les $\mathrm{AD}$, le pasteur étant le modèle et le garant de la morale religieuse, il est indéniable qu'il subisse la sanction disciplinaire en cas d'infraction des principes internes à la dénomination. Cette sanction disciplinaire liée à son divorce d'avec son épouse, est une manière, selon les responsables, de donner une leçon de morale chrétienne à la nouvelle génération de pasteurs. Pourtant, la sanction appliquée aux pasteurs divorcés a des effets socio religieux imprévisibles. Les remous qui ont eu lieu ont perturbé fortement le fonctionnement interne et la cohésion de cette communauté-église.

Au niveau du second cas, le divorce est ordonné par l’époux qui, après plusieurs avertissements remarque une invariance du comportement d’infidélité de l'épouse. Ainsi, il engage la procédure pour obtenir le divorce. C'est ce que décrit ce deuxième cas de divorce développé dans cet encadré ci-dessous.

- Le cas du prophète S. E. de la communauté évangélique MIR de Yopougon

Cette communauté évangélique dite ministère fut créée par S. E. en 2000 à Yopougon. Bien qu'il ait reçu la vocation depuis 1999, S. E. en tant que prophète commença son ministère par une cellule de maison. C'est à partir de cette cellule de maison que son mouvement devint une église autonome en l'an 2000.

Marié depuis quelques années avec G. F., le prophète S. E. vivait paisiblement et en collaboration parfaite avec son épouse dans la gestion de la communauté religieuse dont il est le fondateur. Cependant, dans cette atmosphère de vie harmonieuse G. F. entretenait des relations sexuelles secrètes avec l'un des proches de son époux S. E. Des mois plutard S. E. fut informé de l'acte de son épouse qui, soumise à des interrogations finit par avouer sa faute. Celui-ci lui donna une nouvelle ligne de conduite ferme en l'interdisant de refaire la même erreur. Certes, G.F. s'était engagée de suivre les ordonnances de son époux, mais n’a pas pu respecter son engagement jusqu'au bout. Elle a repris les relations sexuelles avec le jeune disciple de son époux. Cette situation que son époux qualifie d'infidélité devenait de plus en plus récurrente. Cette infidélité récurrente que S. E. ne pouvait plus supporter l'emmena à prendre la décision de rompre d'avec son épouse G. F. Aujourd'hui le prophète S. E. est dans l'attente d'un remariage. Après ce 
divorce le prophète S. E. n’a été objet d'aucune sanction disciplinaire de la part de la communauté. D’ailleurs il demeure toujours le leader et le président fondateur de la communauté MIR. Toutefois, plusieurs membres mécontents ont quitté la communauté pour d’autres horizons. Les autres membres ont accepté le prophète bien qu’il soit divorcé et continuent d’être sous sa tutelle. Ces remous auxquels la communauté fut objet ont ralenti ses activités religieuses et fait régresser son effectif et la portée des ses actions dans l'environnement immédiat.

Yopougon le 20 mars 2016

A l'instar du cas de divorce mentionné plus haut, nous avons des implications aussi bien sur le fonctionnement politique et social de la communauté. Cela montre l'ampleur de la question de divorce de pasteurs dans les communautés chrétiennes évangéliques à Yopougon. Cependant comment les conséquences se manifestent-elles dans ces communautés?

\section{Les effets du divorce des pasteurs chez les évangéliques}

Les effets du divorce sont nombreux, mais nous allons effectuer notre analyse sous deux angles: les implications internes et externes aux communautés évangéliques.

\section{III-1 Effets internes aux communautés évangéliques}

Le divorce, disons-le agit sur le fonctionnement politique et structurel interne de la communauté. Il devient ainsi un facteur de mutation à plusieurs niveaux : individuel, relationnel, comportemental... Au niveau des deux communautés MIR et AD, nous notons plusieurs perturbations.

\section{III-1-1 Les fidèles quittent la communauté-église}

Le départ de fidèles, en tant que premier indicateur vu de l'extérieur, désigne dans ce contexte le mouvement de fidèles chrétiens, de la communauté concernée vers d'autres communautés chrétiennes qui leur semblent plus favorables, ou vers d'autres horizons inconnus. Autrement dit, les fidèles déçus et découragés par l'attitude des pasteurs divorcés quittent les communautés de ces derniers pour d'autres destinations. C'est d'ailleurs ce qu'attestent les propos de l'ancien M. Guéhi que le divorce des pasteurs occasionne de multiples perturbations dans les communautés à tel point que des fidèles quittent l’église.

Ces propos justificatifs montrent comment le divorce de pasteurs agit négativement sur l'effectif et les relations interpersonnelles des fidèles des communautés évangéliques indexées. 
Il se forme de petits groupes pendant ces départs qui deviennent progressivement des cellules de prières et plutard des Eglises autonomes. Une telle métamorphose qui s'opère dans cette situation de crise nous amène à comprendre que des Eglises peuvent naître à partir du divorce d'un pasteur. Ces Eglises qui naissent représentent des ramifications du monde évangélique quelle que soit leur identité (NKP, 2015: 326n).

En outre, le divorce de pasteurs est dans une certaine mesure un facteur de prolifération de nouvelles Eglises indépendantes que sont, entre autres, les ministères et les missions évangéliques ou prophétiques internationales. Les responsables de ces nouvelles églises sont en général des pasteurs autoproclamés dont l'origine remonte de libéralisation religieuse perpétrée en Côte d'Ivoire depuis 1990 dans un contexte de crise socioéconomique (Guiblehon B, 2012: 115). Le concept «international » récurrent dans l'appellation de cette catégorie d'Eglises leur confère un caractère universel et non limité à un pays encore moins à un continent.

Par ailleurs, il existe des personnes qui marquées négativement par le divorce de pasteurs décident de ne plus fréquenter les Eglises en générale et les évangéliques en particulier. Cette catégorie de personnes que les évangéliques appellent "chrétiens rétrogradés" représente, selon eux, un danger pour les Eglises. Abordant dans ce sens le président des jeunes de MIR souligne que " c'est une entité sociale dont les actions tendent à ternir l'image des communautés évangéliques " ${ }^{29}$. Ces propos mettent en évidence la capacité de nuisibilité de ces personnes qui sortent du lot des évangéliques suite à leur marque indélébile due au comportement de divorce de pasteurs. Ces personnes, bien qu'ils soient désormais à l'extérieur de la communauté, gardent toujours un souvenir douloureux et négatif de leur communauté évangélique d'alors.

Ce départ entraine évidemment la chute brutale de l'effectif des Eglises concernées tout en constituant un facteur de croissance d'effectif pour d'autres communautés d'accueil. Le divorce de pasteurs devient alors un élément de régression et de perturbation du fonctionnement social et politique de la communauté. Ces départs sont également l'expression de perte de membres et surtout de responsables qui occupaient une position indispensables à l'évolution de cette communauté.

Z. P. abordant dans cette perspective souligne ceci: "quand un homme de Dieu ${ }^{30}$ divorce les conséquences sont directes et retentissantes dans l'église. L'église se vide à moitié. En générale, les communautés où les pasteurs ont

\footnotetext{
${ }^{29}$ Koré président des jeunes de la communauté MIR.

${ }^{30}$ L'expression "homme de Dieu” désigne tous ceux qui ont pour profession de servir Dieu. Il s’agit notamment des pasteurs, prophètes, évangélistes, apôtres, etc. Ces personnes sont très souvent établies sur des communautés religieuses ou leader de dénomination religieuse.
} 
divorcé, ont disparues après le divorce. Et il y a toujours eu deux groupes : le premier soutien l'épouse, et le second l'époux. Dans d'autres communautés toute l'église se range du coté de l'épouse parce que pour eux, elle est exempte d'erreur. Il existe aujourd'hui des communautés de ce genre à Yopougon. Des pasteurs ont laissé leur église pour aller créer une autre à Cocody ${ }^{31}$ à cause de divorce. Quand ils divorcent dans une communauté ils la quittent pour créer une autre dans un autre quartier où ils sont inconnus $»{ }^{32}$.

A travers les propos de Zouzoua $P$., il résulte que le phénomène de divorce est l'une des sources de dispersion des membres d'une communauté religieuse comme nous l'avons mentionné plus haut. Cependant ce comportement des pasteurs occasionne des divisions et des formations de clans intra-communautaires. Certes, cet acte peut favoriser la création de nouvelles églises mais le pasteur divorcé le fait dans un autre quartier ou une localité où il est moins connu. La méconnaissance de sa personne surtout son passé matrimonial lui permettra d'assoir une nouvelle communauté évangélique pour la continuité de sa profession pastorale. Du coup le divorce de pasteur s’avère un facteur de changement de cadre d'activité religieuse de ceux-ci. Nous pouvons aussi percevoir ce comportement comme une motivation stratégique d'extension communautaire.

Au-delà des mouvements de fidèles, ce comportement des pasteurs évangéliques devient un frein à l'évolution des communautés religieuses de sorte à bouleverser la cohésion des Eglises évangéliques. Ce bouleversement de la cohésion provient des «brisements » des liens de fraternité, ciment de l'unité communautaire et de l'amour tant prôné dan les chapelles évangéliques. C'est une véritable entrave au développement des communautés évangéliques. A partir du divorce de pasteurs, l'image des Eglises évangéliques est ternie. A partir de ces d'union des pasteurs naîtra un désordre relation de sorte à amener certains jeunes évangéliques à une vie sexuelle précoce. Du coup, la confusion gagne progressivement cette couche vulnérable de sort que les normes sociales et religieuses perdront progressivement leur force de lois dans la conscience des fidèles les moins affermis.

Pour rappel, au niveau des Eglises évangéliques pentecôtistes telle que les $\mathrm{AD}$, les pasteurs divorcés sont automatiquement radiés de toutes les communautés du territoire national selon les normes disciplinaires. C'est ce que le pasteur D. essaye de nous traduire par ses propos suivants:

${ }^{31}$ Cocody l'une des Communes du district d'Abidjan où vivent des personnalités et hommes/femmes d'affaires. De ce fait il est considéré comme la Commune des "nantis".

${ }^{32}$ Propos recueilli auprès de pasteur $\mathrm{Z}$. P., leader et fondateur de la MERAD. 
"chez nous les $A D$ le pasteur qui s'adonne au divorce n'est plus un modèle. Donc le collège des pasteurs après délibération sur son cas le radie automatiquement. Nous le faisons pour que cela serve de leçon aussi bien aux fidèles qu'aux jeunes serviteurs de Dieu de notre dénomination ${ }^{33}$.

Ce pasteur met en évidence la sanction disciplinaire appliquée aux pasteurs divorcés chez les $\mathrm{AD}$. Cette application disciplinaire vise à instruire la nouvelle génération de pasteurs et l'ensemble des fidèles. L'attitude des dignitaires des $\mathrm{AD}$ exprime leur volonté d'amener l'ensemble des pasteurs à se conformer aux normes chrétiennes dans tous les domaines de la vie religieuse.

Après l'application des dispositions disciplinaires chez les évangéliques, les conjoints divorcés sont libres dans leur choix, soit de partir ou de rester dans la communauté. Généralement, certains pasteurs divorcés créent leur propre Eglise comme le cas évoqué plus haut avec Y. S, expasteur des AD de Yopougon zone industrielle. Divorcé et radié des AD, Y. S. a créée son Eglise dénommée "église évangélique la maison de la gloire".

L'application de sanction au pasteur divorcé ne se vérifie pas chez les chrétiens de la dénomination MIR. En effet, le cas de divorce que nous avons rencontré concerne le leader, le président fondateur de cette Eglise. En tant que garant de la morale et de la discipline. Après son acte de divorce il n'a connu aucune sanction. Son acte est demeuré dans l'ombre et se discutait entre fidèles réunis en petits groupes. Sa communauté est restée muette même si l'on enregistre le départ progressif de certains membres mécontents. Les membres restés attachés au pasteur divorcé ont résolu de continuer à l'accompagner dans son service religieux. La communauté a connu une régression de taux de fidèles surtout l'effectif des responsables. En dehors des départs de fidèles nous avons constaté que des fidèles ont gardé un souvenir douloureux du divorce de leur pasteur.

\section{III-1-2 "Brisement" de liens de fraternité entre fidèles}

Les brisements de liens et les déchirures du tissu social ainsi que la séparation des liens sociaux s'observent après les départs inattendus. Ce mouvement provoque des ruptures inopinées de liens de fraternités existant entre les membres de la communauté chrétienne. Considérée comme une famille, la communauté chrétienne est perçue comme un tissu dans lequel les membres sont intimement liés. D’ailleurs, c'est ce lien de fraternité qui amène les chrétiens à s'appeler ou à se considérer comme des frères et sœurs. Ceci pour mettre également en évidence cette unité du groupe religieux. Du

\footnotetext{
${ }^{33}$ Pasteur D., l'un des pasteurs de la communauté AD de Yopougon Andokoi.
} 
coup, les séparations inattendues mettent en mal la cohésion de cette entité tout en limitant son rendement. C'est l'expression d'un délitement du lien social entre les membres, surtout la désintégration du système intégrateur au sein même de cette communauté. Il n’est sans doute pas exagéré de parler de dissolution de ce lien social et religieux pourtant essentiel ${ }^{34}$ (Dufaÿ Laurence, 2012) à la survie de toute communauté chrétienne. A partir de ce désordre qui s’installe, des fidèles développent progressivement des comportements d'insoumission au pasteur divorcé. Mieux d'autres utilisent les actes des pasteurs comme motivations suffisantes pour délaisser leur femme qu'ils n'aimaient plus pour d'autre. Ainsi le divorce de pasteur devient un élément excitateur de divorces à répétition dans la communauté chrétienne. Un tel comportement s’inscrit dans la perspective idéologique développée par Boudon. Pour lui l'action collective a des influences sociales sur l'individu. Ainsi le comportement de divorce des pasteurs motive des conjoints à demander le divorce. C'est un comportement qui semble un phénomène de mode dans le milieu évangélique.

En définitive, ce bref parcours de quelques conséquences sociales du divorce des pasteurs dans les communautés évangéliques, nous amène à comprendre que les implications intra communautaires sont nombreuses. Nous les résumons aux perturbations du fonctionnement social, politique, et la destruction de la cohésion des communautés évangéliques.

\section{III-2 Effets externes aux communautés évangéliques}

Les conséquences externes du divorce au cadre religieux sont multiples. Dans cette étude il s'agit des effets sur l'entourage social immédiat des communautés évangéliques et celui des pasteurs victimes et leur progénitures.

Le divorce est quelquefois considéré comme un risque social et une transgression des bonnes mœurs ${ }^{35}$ (Fatou Binétou DIAL, 2001) sociales et religieuses, vue les implications imprévisibles qu'il occasionne dans les ménages et dans la société. Le cas des pasteurs est un fait religieux qui provoque des conséquences diverses, dans l'environnement social des communautés chrétiennes. Ce comportement de ces guides chrétiens donne une autre perception sociale de l'entourage social vis-à-vis de leur personnalité et de leur vocation pastorale.

A cet effet, le cas des $\mathrm{AD}$ de Yopougon zone industrielle s'avère édifiant. Après le départ organisé de l’ex-pasteur Y S, l’environnement social

${ }^{34}$ Dufaÿ Laurence (2012). «Religare à la recherche du lien social », in Diversités et citoyennetés, la lettre de l'IRFAM, N³2, p 4.

${ }^{35}$ Fatou Binétou DIAL (2001). Le divorce, source de promotion pour la femme ?, L'exemple de femmes divorcées de Dakar et de Saint Louis (Sénégal), Colloque international : genre, population et développement en Afrique, Abidjan, p 1. 
de la communauté s’interroge jusqu'aujourd'hui sur les raisons de son exclusion. Le divorce de ce pasteur occupe une place importante dans les débats des jeunes de son quartier. Pendant que les uns s’interrogent sur les raisons d'un tel comportement, d'autres se moquent en généralisant cet acte à l'ensemble des pasteurs des communautés évangéliques.

C'est le même son de cloche dans l'entourage de la dénomination MIR. Plusieurs habitants de la zone proche de cette communauté qui avaient souvent recourt à cette Eglise en cas de problèmes nécessitant un exorcisme ont désormais pris leur distance. Ces habitants ne prennent pratiquement plus part à leurs activités socio religieuses encore moins répondre aux invitations. Partout l'on s’interroge sur la crédibilité du pasteur surtout de sa vocation. Certaines personnes n’hésitent pas à se demander s’il est véritablement un serviteur de Dieu comme il le prétend être.

Pourtant, ces pasteurs étaient vus comme des éclaireurs, des chefs de fil ou des leaders ; ils représentaient même "les Moïse" des communautés religieuses. Cette considération s’explique par le nombre important de personnes de plusieurs horizons qui venaient les consulter. En tant que modèle, référence d'un groupe social et religieux et porte parole de Dieu, ils donnaient des conseils et la direction dans plusieurs domaines: les affaires, le mariage, le travail... A ce titre, ils sont respectés et même imités par des personnes de leur milieu social qui les admirent et les apprécient à leur juste valeur. Elles n’hésitent pas à les présenter en ces termes: "c'est le pasteur de l'église $X^{\prime \prime}$. Par ces actes de courtoisie découlant de la perception et de la considération de l'entourage social, les pasteurs deviennent des personnalités de valeur, des exemples importants dans leurs milieux sociaux spécifiques ${ }^{36}$. Cependant, ils ne sont plus reconnus comme des pasteurs exemplaires mais considérés comme des destructeurs de liens sociaux ${ }^{37}$.

Au-delà du divorce des pasteurs, l'image des communautés évangéliques $\mathrm{AD}$ et MIR est dégradée de sorte que des personnes refusent de les fréquenter encore moins d'y adhérer. Le divorce devient alors un frein à l'adhésion de nouvelles personnes de l'environnement aux communautés évangéliques.

Au niveau du MIR par exemple, avant le divorce du leader, certaines personnes de l'entourage de cette communauté évangélique s’y rendaient régulièrement soit avec des personnes malades, soit avec des personnes tourmentées par des forces mystiques ou des esprits dits maléfiques afin que les responsables prient pour eux. La communauté était devenue un lieu où la

\footnotetext{
${ }^{36}$ Op cit.

${ }^{37}$ Dans cette étude l'expression "liens sociaux" dont nous parlons recouvre deux aspects : d'une part ils sous-entendent les relations qui lient les époux pour qu'ils soient un ; ils supposent également les rapports entre les membres d'un groupe social assurant ainsi la cohésion de l'ensemble.
} 
majorité de ces personnes obtenaient satisfaction à des problèmes. C'est pourquoi elle était regardée comme un hôpital, un lieu de refuge et de consolation. Mais, depuis la rupture du lien conjugal de son leader, ces personnes refusent de s’y rendre.

En outre, le divorce représente pour ces personnes une souillure perpétrée dans un milieu sain où de tels comportements sont interdits. Cette souillure modifie les interactions entre la communauté et la société environnante.

De manière synthétique, voilà comment le divorce des pasteurs agit sur l'entourage des communautés évangéliques. L’acte des pasteurs surtout des leaders donne une autre image de la communauté tout en mettant en cause les enseignements religieux dispensés. Le divorce des pasteurs est de la sorte un risque social et une transgression des bonnes mœurs ${ }^{38}$ (Fatou Binétou DIAL, 2001) religieuses pour les évangéliques et culturelles pour l'entourage. C'est un fait religieux qui occasionne des conséquences dans l'environnement social des communautés évangéliques. Il modifie la perception sociale de l'entourage, par rapport à la personnalité de ces pasteurs divorcés, et au-delà de la communauté religieuse. Ce qui constitue l'un des obstacles à l'évolution de ces communautés dans leur milieu social. Les images des divorcés pasteurs et des communautés sont ternies. Dès lors le divorce devient un frein aux activités religieuses puisqu'il empêche l'adhésion de nouveaux chrétiens.

En plus des implications internes et externes aux communautés évangéliques, il importe de mentionner les effets du divorce sur les pasteurs divorcés et sur leurs enfants.

\section{III-2-1 Situation des pasteurs divorcés}

Le divorce des pasteurs conduit directement à la dissolution de la structure du ménage et amène les enfants à ne plus vivent sous la tutelle directe des deux parents ${ }^{39}$ (Mohamed Lemine, 2002). L’ex-épouse retourne généralement en famille avec les enfants ${ }^{40}$ mineurs, quelque soit son statut social. Mais qu'en est-il des pasteurs?

En effet, le début d'après divorce est toujours douloureux pour les exépouses de pasteurs surtout celles qui sont pasteurs puisqu'elles vivent une

\footnotetext{
${ }^{38}$ Fatou Binétou DIAL (2001). Le divorce, source de promotion pour la femme ?, L'exemple de femmes divorcées de Dakar et de Saint Louis (Sénégal), Colloque international : genre, population et développement en Afrique, Abidjan, p 1.

${ }^{39}$ Mohamed Lemine S. (2002). Etude sur le divorce en Mauritanie, projet IDF-banque mondiale, Mauritanie, p 32.

${ }^{40}$ Au niveau des cas de divorce des pasteurs, il est rare de voir les enfants mineurs habités avec leur père. Les épouses divorcée qui souvent disposent des sources de revenue, pour celles qui ne sont pas pasteurs, arrivent à couvrir une part importante des besoins des enfants, même si le début est toujours douloureux pour les enfants et pour cette dernière.
} 
situation de précarité financière. D’ailleurs, assurer l'éducation et couvrir les besoins des enfants ainsi que la gestion politique, sociale et religieuse efficace de la communauté d'accueil devient une préoccupation épineuse pour ces femmes pasteurs divorcées. Néanmoins, la solidarité de la nouvelle communauté constituée ou celle qui les a accepté leur est très indispensable. Elle leur permet de résoudre plusieurs difficultés.

Pour celles qui ne sont pas pasteur, le soutien parental s'avère une source de réconfort puisqu'elles sont automatiquement acceptées et réintégrées. Cette forme de solidarité spontanée produite par le retour des divorcées dans leur famille respective se rapproche de celle développée par Durkheim Emile (1973) ${ }^{41}$. Pour lui, les actions sociales spontanées que manifeste les membres d'un groupe social envers un membre, étant donné que les individus partagent une même conscience collective ${ }^{42}$ est la solidarité mécanique. Elle se caractérise par la spontanéité manifestée par les autres membres de la société envers le membre en difficulté. Les soutiens sont soit communautaires, soit parentaux. Ainsi certaines ex-épouses de pasteurs arrivent à acquérir des biens divers après le divorce.

C’est pourquoi, lorsque les difficultés financières sont bien réelles et qu'elles se prolongent, elles ne sont pas vécues comme telles par l'ensemble ${ }^{43}$ des ex-épouses de pasteurs divorcées. Au niveau de Yopougon, certaines femmes pasteurs divorcées ont une situation financière plus ou moins améliorée que celles qui ne sont pas pasteurs et qui ne disposent pas de ressources solides, ou qui n'exercent pas une profession stable. Car plusieurs femmes pasteurs divorcés bénéficient de soutiens provenant des membres aussi bien de sa famille, de son réseau social que de sa communauté religieuse d'appartenance.

Certes, les pasteurs divorcés qui demeurent sur leur communauté rencontrent quelques difficultés dans la gestion de la communauté mais, après cette période de trouble ils arrivent à se réorganiser. Ces cas sont beaucoup manifestent dans les communautés néo-pentecôtistes ${ }^{44}$. D’autres divorcés pasteurs arrivent à se repositionner socialement de sorte à améliorer leur situation financière grâce au soutien de leur nouvelle épouse. Ces femmes mettent à la disposition de leur nouveaux époux leurs biens matériels et financiers.

${ }^{41}$ Durkheim Emile (1973). De la division du travail social(1893), PUF, pp 98-101.

${ }^{42}$ Selon Durkheim la conscience collective est un ensemble de croyances et de sentiments communs à la moyenne des membres d'une même population. Ici cette population c'est la parenté, donc la famille d'appartenance de la femme divorcée. Plus cette conscience collective est forte plus le groupe social s'impose à l'individu et règle ses comportements quotidiens. Cette conscience favorise la solidarité spontanée entre les membres de ce groupe social. M. Montoussé et al. (2003 : 4). Voir aussi Danic I. (2006 : 1).

${ }^{43}$ Op cit, p 10.

${ }^{44}$ Il s'agit ici des Eglises dites ministères et missions internationales ou prophétiques. 
Ce mieux être social, matériel et financière qui semble une prospérité est perçue par les fidèles sous leur tutelle comme "une bénédiction matérielle". Dès lors, le divorce peut paraître, sous d'autres aspects comme une voie d'accès au bonheur. Un bonheur résultant de plusieurs actions rationnelles. Cela semble en réalité l'aboutissement de calculs et d'actions rationnelles des leaders. Cette rationalité explicite se rapproche de l'action rationnelle finalité développée par Max Weber (1995) dans son ouvrage Economie et sociétét5.

En effet, pour Weber l'action rationnelle finalité est un instrument tourné vers un but utilitaire et qui implique l'adéquation entre les fins et les moyens. L'acteur social mobilise des stratégies relatives à l'objectif qu'il veut atteindre. C'est ce comportement qui s'observe dans le processus de divorce des pasteurs des Eglises néo-pentecôtistes telle que le MIR. Ces actes obéissent à des stratégies sociales et doctrinales tournés vers la satisfaction maximale de profits et d'intérêts. Cette idéologie de Weber à propos de l'action rationnelle finalité basée sur les calculs a été reprise par Colman et Bourdon ${ }^{46}$ (Voir Tschannen Olivier, 2001).

Colman ${ }^{47}$ estime que les actions des individus sont toujours rationnelles. Pour renchérir cette idée de Colman, Bourdon souligne qu’en plus de la rationalité des actions sociales l'individu est influencé. Autrement dit, le milieu social de l'individu participe ou le conditionne à ces genres d'actions rationnelles. Ainsi, les pasteurs divorcent après un véritable calcul du profit qui en résultera. Le comportement de ces pasteurs évangéliques influence des fidèles et des personnes de leur entourage qui sont amenées et même tentées de divorcer à leur tour. Ces actions qui s’inscrivent dans des contextes sociaux spécifiques s’apparentent à un projet conçu dont la réalisation obéit à un processus bien défini dans le temps.

Des pasteurs issus de ce groupe de divorcés ont tendance à croire que la spiritualité peut être une opportunité d'acquisition de biens, de confort et un moyen de valorisation sociale. Pour eux la religion crée donc des opportunités et le divorce devient le moyen d'accès favorable à la valorisation personnelle. Du coup, le divorce de pasteur semble une source de promotion sociale, un moyen d'enrichissement pour tous ceux qui l'utilise comme stratégie d'acquisition de biens et de satisfaction des attentes. D’ailleurs c'est ce qu'atteste Z. P. par les propos suivants :

«si les pasteurs divorcent c'est parce qu'ils visent un mieux être. Plusieurs divorcent pour se remarier avec des femmes qui possèdent assez de biens matériels et

\footnotetext{
${ }^{45}$ Max Weber (1995). Economie et société (1922), rééd. Coll. Agora, Editions Plon, Paris, pp 36-37.

${ }^{46}$ Voir Tschannen Olivier (2001). Histoire de la sociologie et théorie sociologique, p 4.

${ }^{47}$ Idem
} 
financiers. Ainsi, ils habitent de grandes maisons aux II Plateaux et à Angré, disposent de grosses voitures climatisées qu'elles leurs offrent. La prospérité de ces genres de pasteurs viennent de ces sources là » ${ }^{48}$.

Cette position ci-dessus nous amène à comprendre que le divorce relève dans certains cas de projet personnel, celui d'aspirer à un mieux être avec des partenaires nantis. Du coup nous pouvons dire que le divorce résulte quelquefois d'un choix rationnel pour la satisfaction d'attentes liées à la valorisation personnelle. Au niveau des AD Y. S. a pris ses dispositions puisqu'il était sur la voie de la rupture d'avec sa femme et d'une sanction certaine. Quant au leader de la MIR, bien qu'il soit en train d'entreprendre les démarches pour une nouvelle union, a gardé sa position sur sa communauté.

En somme nous pouvons retenir que les divorces influencent négativement la situation des pasteurs victimes. Cependant, il peut être une source ou un moyen de repositionnement social. Après avoir évoqué la situation des pasteurs divorcés et ses implications spécifiques, qu'en est-il des effets sur leur progéniture?

\section{III-2-2 Effets du divorce sur les enfants de pasteurs divorcés}

Généralement le divorce est un évènement dramatique ${ }^{49}$ (Brusa Alberto, 2009) pour les enfants des conjoints dont le lien d'union est rompu. A travers les deux communautés évangéliques cibles de Yopougon où s'effectue cette étude, il existe trois cas de figures au niveau des enfants de pasteurs divorcés.

Dans le premier cas qui est récurrent, les enfants sont sous la garde de l'ex-épouse, donc la mère de ceux-ci. Elle se charge alors de leur socialisation, de leur scolarité et de leur éducation, en accord avec son exépoux. Pour le second cas, les enfants restent sous la garde du pasteur divorcé. Dans ce cas, les plus jeunes pleurent parfois nuitamment en réclamant la présence de leur "maman". Chez d'autres pasteurs divorcés, après le remariage, les rapports enfants et nouvelle épouse sont entachés de tensions et parfois de discussion vives durant la première année de cohabitation. A partir de ces altercations des enfants manifestent le désire de quitter le domicile conjugal. Si le pasteur remarié ni prend garde ces enfants mécontents regagnent définitivement l'un des membres de la famille soit du père, soit de la mère. Ce changement de domicile qui n'est pas toujours du goût du père, crée des tensions dans les rapports entre parents et enfants.

\footnotetext{
${ }^{48}$ Propos recueilli auprès du pasteur Z. P., président fondateur de la communauté évangélique la Mission Evangélique des RAchetés de Dieu (MERAD).

${ }^{49}$ Brusa Alberto C. (2009). « Le divorce et les enfants à bas âge » in ERES/Spirale $\mathrm{N}^{\circ} 49$, p 4.
} 
Le troisième cas de figure qui s’observe dans cette désunion dans les couples de pasteurs, c'est qu'après la rupture du lien conjugal les enfants sont automatiquement placés sous la garde de la mère du pasteur. Cette dernière, soutenue financièrement par le pasteur, assure la socialisation de ceux-ci.

En dehors de ces observations, ajoutons que des enfants de pasteurs divorcés rencontrent plusieurs difficultés dont celui relevant des domaines affectif, éducationnel et financier. Ce qui conduit les jeunes filles adolescentes à la sexualité précoces et aux grossesses indésirées. Quant aux garçons, les moins endurants abandonnent l'école pour des métiers en vue de se prendre en charge. A cet effet, Archambault (2002) souligne que le divorce de conjoints est associé à une réussite scolaire plus faible chez les enfants ${ }^{50}$. D'autres par contre font de longues études à l'aide des soutiens financiers et matériels des deux parents. Il s'agit surtout ici de parents qui pourvoient régulièrement aux besoins des enfants et qui les rendent visite. A ceux là, le divorce n'empêche pas d'atteindre leur objectif au niveau des enfants. Ils mettent des moyens important à la disposition des enfants pour leur réussite scolaire.

Sur le plan religieux, le divorce de parents pasteurs constitue un frein à l'engagement religieux de leurs enfants surtout quand ils sont sous la garde d'une tierce personne. Quelques uns sortent définitivement de la morale chrétienne, puisqu'étant marqué par cette rupture spontanée du lien de mariage des parents.

En définitive, nous pouvons retenir que le divorce de pasteurs crée plusieurs mutations significatives dans la socialisation de leurs enfants. Il devient donc un facteur de modification comportemental et de déviance pour ces jeunes gens. D'ailleurs Togliatti Malagoli et al. $(2005)^{51}$ inscrivent leur pensée dans cette perspective. Pour eux le divorce est l'un des facteurs de risque ou d'obstacle au développement des enfants. Le cas des deux communautés évangéliques de Yopougon montre bien que le divorce perturbe les enfants sur les plans : financier, social, religieux, éducatif, etc. Au-delà ces enfants peuvent se constituer en danger potentiel pour la société. Ils n’ont plus de repère moral et d'orientation parentale véritable.

\section{Conclusion}

Au terme de cette étude nous pouvons retenir que le premier axe de notre réflexion a consisté à la compréhension des fondements doctrinaux du

\footnotetext{
${ }^{50}$ Archambault Paul (2002). «Séparation et divorce : quelles conséquences sur la réussite scolaire des enfants » in population et sociétés, N³79, INED, p 1.

${ }^{51}$ Togliatti Malagoli et al. (2005). « Les enfants du divorce comme protagonistes actifs de la séparation conjugale » in Cahiers critiques de thérapie familiales et de critiques de réseaux, De Boeck Université, p 2.
} 
divorce. Le divorce étant une résultante évidente du mariage, nous avons analysé brièvement les fondements religieux du mariage. Il ressort de ces analyses que le mariage chez les évangéliques est un acte hétérosexuel, c'està-dire qu'il concerne uniquement l'homme et la femme. Cette disposition qui relève des prescriptions bibliques et doctrinales est le modèle matrimonial défendu par l'Eglise jusqu'aujourd'hui. En effet, l'union entre l'homme et la femme préfigure la relation sacrée entre Dieu et le peuple d'Israël d'une part, et la relation permanente entre Jésus-Christ (l’époux) et l'Eglise (l’épouse). Cette relation est sacrée et permanente puisqu'elle représente une alliance indestructible, du point de vue biblique. De ce fait, le lien entre les conjoints issu du mariage est indissoluble. Car il symbolise cette alliance divine et sacrée qui perdure entre les époux quelque soit les raisons évoquées. Du coup, le divorce devient le symbole de la rupture de cette alliance sacrée et permanente. A cet effet, Georges Balandier (1996) précise que:

"le sacré se dédouble en un sacré pur, garant de l'ordre, du bien, des valeurs, et un sacré impur, générateur du désordre, du mal, de la transgression. En sommes le sacré se trouve là où s'effectue l'affrontement de l'ordre et de désordre, du bien et du mal, des valeurs contraignantes et de la transgression subversive. C'est une ligne de front » ${ }^{52}$ (Balandier Georges, 1996).

Détruire le lien sacré c'est semer le désordre source de mal et de transgression entre les conjoints et Dieu, entre les conjoints et l'Eglise, et entre les conjoints eux-mêmes.

Le deuxième axe a mis en évidence les facteurs justificatifs du divorce des pasteurs chez les évangéliques. Nous retenons de cette analyse que le divorce des pasteurs s'explique par plusieurs facteurs dont les plus essentiels sont: les tensions et les disputes récurrentes dans le couple ; l'infidélité continue des époux; le manque de pardon et d'acceptation réciproque des conjoints en cas d'erreur, la fuite subtile de l'un des conjoints du foyer, etc. Bien qu'ils soient des guides et des bergers humains, les pasteurs succombent à ces situations et détruisent le lien sacré de leur mariage.

Le troisième axe concerne les conséquences issues de divorce de pasteurs. Cette section de l'étude a relevé qu'à l'intérieur des communautés s’observe des désordres, des divisions et le départ de certains fidèles vers d'autres horizons. De ces mouvements naissent des groupes de prières qui deviennent des Eglises par les actions d'évangélisation de ses membres. Quand aux pasteurs divorcés, ils sont soit excommuniés, soit acceptés selon

${ }^{52}$ Balandier Georges (1996). “le sacré par le détour des sociétés de la tradition” in Cahiers internationaux de sociologie, vol 100, version électronique consulté le 29/04/2015, PUF, Paris, p 9 
les types de communauté chrétienne. A l'extérieur la situation des pasteurs divorcés, déçus et découragés devient objet de critique et de débats.

Toutefois, il importe souligner que, pour des pasteurs le divorce représente une stratégie de positionnement social, de valorisation de leur personnalité et d'acquisition de richesse. Ainsi ce comportement devient un moyen pour la réalisation de désirs personnels.

\section{References:}

1. Ako A. G. (2013). Fiançailles, mariage et divorce en milieu chrétien évangélique ivoirien: cas des assemblées de Dieu de Yopougon Andokoi, mémoire de master I à l'Université Houphouët Boigny de Cocody, Abidjan, 75 p.

2. Aman K. L. (2006). L'Eglise Méthodiste unie-Côte d'Ivoire et le mariage, mémoire de Licence en théologie, ISTHA, Abidjan, 48 p.

3. Atsain A. J. P. et al. (1997). Attitude des Ivoiriens face aux divorce, le cas de Yopougon toit-rouge, Mémoire de Licence, Université d'Abidjan, IES,72 p.

4. Archambault Paul (2002). «Séparation et divorce: quelles conséquences sur la réussite scolaire des enfants » in population et sociétés, N³79, INED, 4 p.

5. Balandier Georges (1996). "Le sacré par le détour des sociétés de la tradition" in Cahiers internationaux de sociologie, vol 100, version électronique consulté le 29/04/2015, PUF, Paris, pp 5-12.

6. Bale M. (1988). Le mariage en Côte d'Ivoire : la problématique d'un conflit entre la tradition le et modernisme : étude de cas à Abidjan, Mémoire de Maîtrise, Université d’Abidjan, IES, 96 p.

7. Billiez Jacqueline \& Millet Agnès (2015). Représentations sociales : trajets théoriques et méthodologiques, S. Ed, University of Grenoble, $17 \mathrm{p}$.

8. Brusa Alberto C. (2009). «Le divorce et les enfants à bas âge » in ERES/Spirale-1 N49, S Ed, SL, pp 25-31.

9. Chimoun Mosé (2004). Transgressions des tabous sexuels dans les romans féministes de l'Afrique de l'Ouest, du centre et du monde germanophone in Langues et Littératures, Université Gaston berger de Saint-Louis, N8, Sénégal, pp 63-76.

10. DIAL Fatou Binétou (2001). Le divorce, source de promotion pour la femme ?, L'exemple de femmes divorcées de Dakar et de Saint Louis (Sénégal), Colloque international: genre, population et développement en Afrique, Abidjan, 15 p.

11. Dufaÿ Laurence (2012). « Religare à la recherche du lien social », in Diversités et citoyennetés, la lettre de l’IRFAM, N³2, 30 p. 
12. Durkheim Emile (1973). De la division du travail social(1893), PUF, Paris, 416 p.

13. Fatou Binétou DIAL (2001). Le divorce, source de promotion pour la femme ?, L'exemple de femmes divorcées de Dakar et de Saint Louis (Sénégal), Colloque international : genre, population et développement en Afrique, Abidjan, 15 p.

14. Guiblehon Bony (2012). Les jeunes et le marché de la spiritualité pentecôtiste en Côte d'Ivoire, in European Scientific Journal, October Edition vol. 8, No.24 pp 115-135.

15. Kosongo M. P \& Kinable J. (2010). Enfants de la rue, phénomène pluriel et complexe à Lubumbaschi, RDC Congo, Acte du Colloque international francophone «Complexité 2010 ». La pensée complexe : défis et opportunités pour l'éducation, la recherche et les organisations-Lille(France) mercredi 31 mars et jeudi $1^{\mathrm{er}}$ avril 2010, $12 \mathrm{p}$.

16. Max Weber (1995). Economie et société (1922), rééd. Coll. Agora, Editions Plon, Paris.

17. Murray John (1992). Le divorce : les données bibliques, Collection Alliance, les Editions Sator, Grande Bretagne, 144 p.

18. Mohamed Lemine S. (2002). Etude sur le divorce en Mauritanie, projet IDF-banque mondiale, Mauritanie, 49 p.

19. Oliver de Sardan JP. (2003). L'enquête socio-anthropologique de terrain : synthèse méthodologique et recommandations à usage des étudiants, études et travaux $\mathrm{N}^{\circ} 13$, version électronique consultée le 25/04/2015, LASDEL, Niamey, 59 p.

20. Piras Mauro T. (2004). Les fondements sociaux de l'agir normatif chez Durkheim et Weber : le rôle du sacré in Archives des Sciences Sociales des Religions, 127, pp 139-166.

21. Sebo Kouami Félicien (2014). Faut-il un mariage pour tous ? Un défi pastoral pour l’Eglise en Afrique, question controversées, les Editions du CERAP, Abidjan, 110 p.

22. Tschannen Olivier (2001). Histoire de la sociologie et théorie sociologique, Support de cours, S. Ed, Université de Fribourg, 28 p.

23. Togliatti Malagoli et al. (2005). «Les enfants du divorce comme protagonistes actifs de la séparation conjugale » in Cahiers critiques de thérapie familiales et de critiques de réseaux, $\mathrm{S} \mathrm{Ed}$, De Boeck Université, pp 135-156.

24. W Doise (1990). « Les représentations sociales » in $R$ Ghiglione, $C$. Bonnet, J F Richard, Traité de psychologie cognitive, Tome 3, Ed. Dunod, SL, pp 110-174. 